such as that in the Mare Imbrium could be caused by an object roughly 20 millionths of the mass of the Moon buried $50 \mathrm{~km}$ below the surface. The obvious explanation, that the hidden bodies are the remains of asteroids which excavated the ringed maria, does not seem to be feasible. The object under Imbrium is equivalent to a nickel-iron sphere $100 \mathrm{~km}$ across and centred at a depth of $50 \mathrm{~km}$, yet a bombarding asteroid would have blown itself to pieces at the high speed of impact. Others have skirted the problem by saving that the bombardment hypothesis could still be correct if the maria were excavated by low velocity asteroids, which might have left some debris behind. For these hypothetical asteroids to have had a sufficiently small velocity relative to the Moon, they must originally have been in Earth orbit. An alternative explanation is that the maria contain lava flows denser than the surrounding rocks. Gilvarry has revealed his ingenuity once again with an explanation in terms of his hypothesis that there were once copious amounts of water on the Moon (Nature,221, 732; 1969), and Goudas has the elegantly simple explanation that the maria are at a higher altitude than previously thought (Nature, 220, 1111; 1968).

\section{SPACE RESEARCH}

\section{Experiments in Satellites}

\section{from a Correspondent}

IT was a pity that the meeting at the Royal Society on April 22 was restricted solely to United Kingdom experiments on NASA and ESRO satellites. Although some useful information was exchanged between rarious British groups working in the field, many of the experiments discussed were so obviously part of a wider European or American programme of research as to make the discussion at times fragmentary.

One subject that attracted attention was the interaction of the magnetic fields of the Sun and the Earth. A contribution from $\mathrm{Mr} \mathrm{R}$. M. Freeman, Mr K. Norman and Professor A. P. Willmore, of University College, London, described measurements on plasmas in space out to distances of about 20,000 kilometres. Rapid changes were found in the density of charged particles near the boundary of the Earth's magnetic field, thus corroborating and extending previous measurements. Dr P. C. Hedgecock, of Imperial College, London, described the large fluctuations in magnetic field that he observed in the region where the magnetic fields of the Earth and the Sun interact most clearly. He also mentioned the results of a French group working on the same satellite, in which has been found an anticorrelation between the number of particles and the field strength in this region of space-which means more particles at lower field.

The study of $\mathrm{X}$-rays and gamma rays from outside the solar system is producing some promising results. Professor G. W. Hutchinson, of the University of Southampton, presented some preliminary data recorded on the OGO-5 satellite using a spark chamber-a sophisticated piece of equipment for use in a satellite. His group has studied $50 \mathrm{MeV}$ gamma rays and has succeeded in measuring the direction of arrival of individual photons to an accuracy of about three degrees. The implication is that a strong flux of gamma rays is coming from the direction associated with the galactic plane.
The changing chemical composition of the ionosphere was discussed by Dr G. L. Wrenn, of University College, London, who reported experiments carried out in the upper ionosphere on the satellite Explorer 31. During the past five years, the concentration of helium in the ionosphere seems to have been steadily decreasing, but Dr Wrenn has found that this trend has now reversed and concentrations of helium are once more increasing. Another set of measurements on the satellite also demonstrated rather well how the charged particles in the ionosphere seem to move in strata parallel and perpendicular to the Earth's magnetic field. This phenomenon derives from the potential barrier to motion perpendicular to the Earth's magnetic field.

\section{MAGNETIC DISTURBANCES \\ Regular Pulsations in Orbit}

\section{from our Magnetosphere Correspondent}

REMARKABLy regular pulsations have been found in the magnetograms from the geostationary satellite ATS 1 (Cummings et al., J. Geophys. Res., 14, 778; 1969). The pulsations are nearly monochromatic and usually nearly linearly polarized, the polarization ellipse being almost perpendicular to the steady magnetic field. The sensitivity of the magnetometer is about $1 \gamma\left(10^{-5}\right.$ gauss $)$ and amplitudes up to $10 \gamma$ have been observed. The pulsations are observed only during magnetically quiet periods $(K p<3)$ and chiefly between 0400 and 1600 local time. With these conditions, they are detectable for a few per cent of the time.

The pulsation events lasted for up to 460 minutes and the pulsation period usually varied little during an event. A histogram for the periods shows two groups, one centred on 190 seconds and the other on 102 seconds. The longer period occurred in very quiet conditions and never during local night-time. Cummings et al. suggest that these pulsations are related to the "giant micropulsations", observed on the ground near the auroral zones, discussed in the $1930 \mathrm{~s}$, and this seems plausible. Following earlier suggestions, their notion is that quasi-transverse hydromagnetic standing waves can occur for which the disturbance is concentrated near one line of force, in line with the observed localization of giant micropulsations. When the disturbance in the magnitude of the field is neglected, so that the disturbance is purely in direction, the hydromagnetic equations for weak disturbances simplify in such a way that only spatial variation along a field line is involved so that normal modes can be calculated for a field line. Cummings et al. have carried out normal mode computations for the first six harmonics and several density models. They point out that the odd harmonics have no magnetic disturbance at the equator and that it is most plausible that the pulsations they observed on ATS 1 are the second harmonic. For giant micropulsations, the phase relations between conjugate points also require even modes.

The periods agree satisfactorily with the assumption that the plasmapause is outside the synchronous orbit when the longer period is observed; the plasmapause is known to move out in the very quiet conditions corresponding to the longer period. The computations also show that the amplitude should be an order of magnitude larger at the reflecting surface than at the equator, while the pulsations observed at the ground seldom 